\title{
IMPAIRED ENDOTHELIUM-DERIVED HYPERPOLARIZING FACTOR-MEDIATED RELAXATION IN CORONARY ARTERIES BY COLD STORAGE WITH UNIVERSITY OF WISCONSIN SOLUTION
}

Guo-Wei He, MD, PhD

Cheng-Qin Yang, MD

\begin{abstract}
Objectives: University of Wisconsin solution is widely used to preserve organs for transplantation, but its effect on the individual endotheliumderived relaxing factors has not been studied. This study was designed to examine the effect of cold storage of the heart with University of Wisconsin solution on relaxation mediated by the endothelium-derived hyperpolarizing factor (EDHF). Methods: Porcine coronary artery rings were studied in organ chambers. Relaxation in response to the EDHFs stimuli bradykinin and $\mathrm{A} 23187$ in $\mathrm{U} 46619$ (30 $\mathrm{nmol} / \mathrm{L})$-induced precontraction after incubation with University of Wisconsin solution (either at $37^{\circ} \mathrm{C}$ in the oxygenated organ chamber or at $4^{\circ} \mathrm{C}$ in a refrigerator for 4 hours) was compared with the control. Results: During the incubation, the coronary tone initially increased transiently $(4.8 \pm 0.8 \mathrm{gm})$ and was subsequently reduced by $10.9 \pm 1.2 \mathrm{gm}$. Under both normothermia and hypothermia, after the incubation, the relaxation mediated by EDHF significantly decreased (under normothermia: from $68.7 \% \pm 10.2 \%$ to $32.1 \% \pm 8 \%, n=7, p=$ 0.001, for bradykinin and from $79.9 \% \pm 8.4 \%$ to $56.9 \% \pm 8.5 \%, n=7, p=$ 0.01, for A23187; under hypothermia and hypoxia: to $18.9 \% \pm 5.6 \%, n=9$, $p=0.0005$, for bradykinin and $52.7 \% \pm 7.5 \%, n=9, p=0.03$, for A23187). The incubation at normothermia also impaired the coronary smooth muscle contractility to $\mathrm{U} 46619$, but this contractility was preserved by cold storage. Conclusions: During cold storage, University of Wisconsin solution impairs the endothelium-dependent relaxation mediated by EDHF in the coronary circulation. This effect exists after the storage for at least 1 hour. (J Thorac Cardiovasc Surg 1998;116:122-30)
\end{abstract}

$D^{-1}$ uring the preservation period of the donor organ, the vascular endothelium is in direct contact with the preservation solution. The effect of organ preservation solutions on endothelium is therefore an important issue in organ transplantation surgery. In fact, such effect has been investi-

From the Division of Cardiothoracic Surgery and Cardiovascular Research Laboratory, Department of Surgery, University of Hong Kong, Grantham Hospital, Hong Kong.

This study was supported by a Hong Kong Research Grants Council Grant (338/048/0001), the University of Hong Kong Committee of Research and Conference Grants (337/048/ 0018 and 335/048/0079), and University of Hong Kong Grants (014/048/9602 and 344/048/0001).

Received for publication June 6, 1997; revisions requested Oct. 14, 1997; revisions received Feb. 9, 1998; accepted for publication Feb. 17, 1998.

Address for reprints: Professor Guo-Wei He, Chair of Cardiothoracic Surgery, University of Hong Kong, Grantham Hospital, 125 Wong Chuk Hang Rd., Aberdeen, Hong Kong.

Copyright (C) 1998 by Mosby, Inc.

$0022-5223 / 98 \$ 5.00+0 \quad \mathbf{1 2} / \mathbf{1} / \mathbf{8 9 8 0 1}$ gated in several recent studies. ${ }^{1-10}$ It has been suggested that University of Wisconsin solution (UW) reduces the endothelium-dependent relaxation $^{8,10,11}$ or causes endothelial dysfunction. ${ }^{12}$

Endothelium plays an important modulatory role on vascular tone. Endothelium-dependent relaxation is known to be the effect of a variety of different endothelium-derived relaxing factors (EDRFs). These are endothelium-derived nitric oxide (EDNO), epoprostenol (prostacyclin $\left[\mathrm{PGI}_{2}\right]$ ), and endothelium-derived hyperpolarizing factor (EDHF). ${ }^{6,7,9}$ Because of the complexity of the endothelial function, it is necessary to study the effect of UW on individual EDRFs to challenge the "perfect" method to preserve the endothelium. However, previous studies did not distinguish the effect of the individual EDRFs, and the effect of UW on the EDHF-mediated endothelial function has never been studied.

Since $\mathrm{K}^{+}$at high concentrations (hyperkalemia) is a key component in organ preservation solutions for 
transplantation or in cardioplegic solutions, we have performed a series of experiments to examine the effect of hyperkalemia on endothelial function. Our previous studies ${ }^{3,4}$ have demonstrated that when only cyclooxygenase pathway is blocked by indomethacin (INN: indometacin), the endothelium-dependent (composed of both EDNO and EDHFmediated) relaxation is not altered by exposure to $\mathrm{K}^{+}$up to $4 \frac{1}{2}$ hours, ${ }^{3,4}$ and this is in agreement with studies by other investigators. ${ }^{2}$ Furthermore, our most recent studies have demonstrated that when EDNO and $\mathrm{PGI}_{2}$ pathways are inhibited, the residual relaxation (mediated by the third component (EDHF) is significantly reduced by exposure to hyperkalemia $\left(\mathrm{K}^{+}\right.$concentration of 20 to $50 \mathrm{mmol} /$ L). ${ }^{6,7}$ The mechanism of the impaired EDHFmediated relaxation after exposure to hyperkalemia is related to the inhibition of $\mathrm{K}^{+}$channels and prolonged depolarization of the smooth muscle membrane potential. ${ }^{6,9}$

In $\mathrm{UW}$ the concentration of potassium $\left(\mathrm{K}^{+}\right)$is as high as $125 \mathrm{mmol} / \mathrm{L}$. The extremely high $\mathrm{K}^{+}$concentration in UW is one of the major concerns with regard to endothelial preservation. ${ }^{10,13}$ However, the composition of UW is complex and the effect of this organ preservation solution may therefore be complicated by its multiple components. The effect of the storage of organs with UW on EDHFmediated relaxation is unclear. It is unknown whether other components in this solution would prevent the organ from the alteration of EDHFmediated relaxation owing to its extremely high $\mathrm{K}^{+}$ concentration.

The present study was designed to answer these questions. We examined the effect of UW (1) under normothermia without the combined effect of ischemia and temperature change and (2) after hypothermic storage combined with hypoxia as in the clinical setting.

\section{Methods}

Coronary arteries were obtained from porcine hearts that were harvested in a local abattoir. Immediately after the hog (either sex) was killed, the heart was rapidly removed, placed in a container filled with Krebs solution at $4^{\circ} \mathrm{C}$, and transferred to the laboratory. Epicardial coronary arteries were dissected free from the surrounding connective tissue, cut into $3 \mathrm{~mm}$ long rings, and mounted on a pair of stainless steel wires in organ chambers ${ }^{6,14}$ filled by Krebs solution at $37^{\circ} \mathrm{C}$. The Krebs solution had the following composition (in millimoles per liter): $\mathrm{Na}^{+} 144, \mathrm{~K}^{+} 5.9, \mathrm{Ca}^{2+} 2.5, \mathrm{Mg}^{2+} 1.2, \mathrm{Cl}^{-}$128.7, $\mathrm{HCO}_{3}{ }^{-} 25, \mathrm{SO}_{4}{ }^{2-} 1.2, \mathrm{H}_{2} \mathrm{PO}_{4}^{-} 1.2$, and glucose 11 . The solution was aerated with a gas mixture of $95 \%$ oxygen and $5 \%$ carbon dioxide at $37^{\circ} \mathrm{C}$.

A previously described organ-chamber technique $e^{6,14}$ was used to normalize vascular rings under a physiologic pressure with a computerized program (VESTAND 2.1 by Yang-Hui He, Princeton University, N.J.). ${ }^{15}, 16$

The endothelium was carefully preserved by cautiously dissecting and mounting the rings. ${ }^{6,15,16}$ To examine the endothelium dependence of the relaxation, in some rings we removed the endothelium mechanically by using a fine wood stick moistened with Krebs solution to rub the intima of the rings gently. The tension development was recorded by a recording system composed of a Grass FT 03 transducer, a Grass preamplifier, and a personal computer by using the PolyView program (Grass Instrument Division, West Warwick, R.I.). Six organ-chamber arrangements were run concurrently.

\section{Protocol}

\section{Exposure to UW under normothermia}

ConTrol. All rings were equilibrated for 30 minutes before and after normalization. Indomethacin $(7 \mu \mathrm{mol} / \mathrm{L})$, a cyclooxygenase inhibitor, and $N^{\mathrm{G}}$-nitro-L-arginine ( $\mathrm{L}$ NNA, $300 \mu \mathrm{mol} / \mathrm{L}$ ), a nitric oxide synthase inhibitor, were added into the bath for 30 minutes. U46619 (30 nmol/L) was then added into the organ chamber to contract the rings. When the contraction reached a stable plateau (usually 10 minutes), cumulative concentration-response curves to receptor-mediated agonist bradykinin (BK) or non-receptor-mediated stimulus calcium ionophore A23187 were established. The concentrations were -10 to $-6.5 \log \mathrm{mol} / \mathrm{L}$ for BK and A23187.

The rings were then repeatedly washed and the following protocol was applied.

UW EXPOSURE. When the tension of the rings returned to the previous level, the bath solution was changed to UW. To isolate the effect of UW from ischemia, the UW was continuously bubbled with a mixture of $95 \%$ oxygen and $5 \%$ carbon dioxide at $37^{\circ} \mathrm{C}$. The incubation time to the UW was 4 hours. During the incubation, the tension of the coronary rings was continuously recorded.

After incubation for 4 hours, the rings were repeatedly washed again for 30 minutes and L-NNA and indomethacin were added for another 30 minutes. This protocol was then repeated.

Time CONTROL. To exclude the effect of time (4 hours) on endothelial function, rings were left in the organ chamber for 4 hours in Krebs solution, instead of UW, and the EDHF-mediated relaxation to $\mathrm{BK}(n=6)$ or A23187 $(n=8)$ was repeated again.

2. Cold storage with UW. Coronary arteries were immersed in UW at $4^{\circ} \mathrm{C}$ for 4 hours without oxygenation. The artery was then suspended in the organ chamber and repeatedly washed with Krebs solution. The solutions in the organ chamber were continuously aerated with a mixture of $95 \%$ oxygen and $5 \%$ carbon dioxide for the subsequent experiment. After equilibration for 1 hour, the normalization procedure was performed and the rings were equilibrated again as mentioned earlier. The relaxation to BK $(n=9)$ or A23187 $(n=9)$ in U46619-induced precontraction was tested in the presence of indomethacin and L-NNA. The results were compared with the control 
(stored in Krebs solution for 4 hours at $4^{\circ} \mathrm{C}$, instead of UW, before the experiment).

3. Contractility after exposure to UW. In normothermic experiments, after UW incubation, the contraction to U46619 (30 nmol/L) was reduced (see Results). Therefore, in 10 rings, after exposure to UW for 4 hours, the concentration-contraction curve to U46619 was established. The contractility (expressed by the maximal contraction force and the $\mathrm{EC}_{50}$ ) was compared with that in the control rings.

The composition of UW is as follows: $\mathrm{Na}^{+} 29 \mathrm{mEq} / \mathrm{L}, \mathrm{K}^{+}$ $125 \mathrm{mEq} / \mathrm{L}, \mathrm{Mg}^{2+} 5 \mathrm{mEq} / \mathrm{L}$, pentafraction $50 \mathrm{gm} / \mathrm{L}$, lactobionic acid $35.83 \mathrm{gm} / \mathrm{L}$, raffinose $17.83 \mathrm{gm} / \mathrm{L}$, adenosine 1.34 $\mathrm{gm} / \mathrm{L}$, allopurinol $0.136 \mathrm{gm} / \mathrm{L}$, and total glutathione 0.922 $\mathrm{gm} / \mathrm{L}$. The $\mathrm{pH}$ of the solution is 7.4 at room temperature.

The effective concentration of the contraction or relaxation agent that caused $50 \%$ of maximal contraction (or relaxation) was defined as $\mathrm{EC}_{50}$. The $\mathrm{EC}_{50}$ was determined from each concentration-relaxation curve by a logistic, curve-fitting equation: $\mathrm{E}=\mathrm{MA}^{\mathrm{P}} /\left(\mathrm{A}^{\mathrm{P}}+\mathrm{K}^{\mathrm{P}}\right)$, where $\mathrm{E}$ is response, $\mathrm{M}$ is maximal contraction (or relaxation), $\mathrm{A}$ is concentration, $\mathrm{K}$ is $\mathrm{EC}_{50}$ concentration, and $\mathrm{P}$ is the slope parameter. ${ }^{14}$ From this fitted equation, the mean $\mathrm{EC}_{50}$ value \pm standard error of the mean was calculated for each group.

Statistical analysis. When the comparison was made in the same ring for the maximal response (either relaxation or contraction) in time-control experiments or under normothermia, the paired $t$ test was used. The maximal response (either relaxation or contraction) in two groups of different rings, such as in the cold storage experiments, was compared by the unpaired $t$ test.

Drugs. Drugs used and their sources were BK, A23187, (L-NNA), and indomethacin (Sigma, St. Louis, Mo.), and U46619 (Cayman Chemical, Ann Arbor, Mich.). UW was purchased from Du Pont Merck Pharmaceutical Co. (Wilmington, Del.). L-NNA (dissolved in distilled water) and indomethacin (dissolved in ethanol) were stored at $4^{\circ} \mathrm{C}$. The solution of U46619 was held frozen until required.

\section{Results}

The coronary tension during exposure to UW. Under normothermia in the organ chamber, during incubation with UW, the coronary tone showed twophased changes. It transiently increased during the first 7.5 minutes and reached the peak $(4.8 \pm 0.8 \mathrm{gm})$ at 3.5 minutes, followed by a decrease after 8 minutes. Forty minutes later, the decrease of tone reached more than $95 \%$ of the lowest value $(-10.9 \pm 1.2 \mathrm{gm}$ from the baseline) and remained at this low tone for the subsequent incubation period (Fig. 1).

Under cold storage, the coronary arteries were at resting status in a refrigerator and there was no attempt to record the tension.

The effect of UW on EDHF-mediated endothelium-dependent relaxation

Normothermia

BK. UW exposure reduced the BK-induced relaxation from $68.7 \% \pm 10.2 \%$ in the control group to $32.1 \% \pm 8 \%(n=7,95 \%$ confidence interval: $0.04 \%$ to $1.0 \%, p=0.001$, paired $t$ test) (Fig. $2, a$ ). In the control rings, the $\mathrm{EC}_{50}$ was $-7.82 \pm 0.1 \mathrm{log}$ $\mathrm{mol} / \mathrm{L}$. After UW treatment, in some rings, the relaxation was almost abolished and therefore the $\mathrm{EC}_{50}$ value could not be calculated.

A23187. Similarly, UW exposure reduced the A23187-induced relaxation from $79.9 \% \pm 8.4 \%$ to $56.9 \% \pm 8.5 \%(n=7,95 \%$ confidence interval: $-0.05 \%$ to $1.0 \%, p=0.01$, paired $t$ test) (Fig. 2, $b$ ). In the control rings, the $\mathrm{EC}_{50}$ was $-7.07 \pm 0.09 \mathrm{log}$ $\mathrm{mol} / \mathrm{L}$. After UW treatment, the $\mathrm{EC}_{50}$ value increased to $-6.80 \pm 0.16 \log \mathrm{mol} / \mathrm{L}$ (95\% confidence interval: -0.004 to $0.96 \log \mathrm{mol} / \mathrm{L}, p=0.04)$.

In time-control arteries, there was no significant difference in the first and second relaxation induced by either BK $(76.7 \% \pm 7.3 \%$ vs $85.4 \% \pm 5.4 \%, n=$ $6, p=0.4$, paired $t$ test $)$ or A23187 $(81.3 \% \pm 7.9 \%$ vs $68.4 \% \pm 12.1 \%, n=8, p=0.13$, paired $t$ test). The contraction induced by U46619 (30 nmol/L) did not change in the BK group $(10.1 \pm 0.7 \mathrm{gm}$ vs $10.8 \pm$ $1.1 \mathrm{gm}, p=0.26$ ), although it slightly increased in the A23187 group $(13.8 \pm 1.2$ vs $15.4 \pm 1.2 \mathrm{gm}, n=$ $8,95 \%$ confidence interval: 0.8 to $1.0, p=0.001$, paired $t$ test).

Cold storage with $U W$

BK. After UW exposure, the BK-induced relaxation was $18.9 \% \pm 5.6 \%(n=9, p<0.001$, compared with the control of $76.7 \% \pm 7.2 \%, 95 \%$ confidence interval: $0.7 \%$ to $10.8 \%$, unpaired $t$ test, Fig. 3,a). In two of the arteries, the relaxation was abolished so the $\mathrm{EC}_{50}$ value could not be calculated.

A23187. Similarly, UW exposure reduced the A23187-induced relaxation from $80.7 \% \pm 5.5 \%$ $(n=15)$ to $52.7 \% \pm 7.5 \%(n=9,95 \%$ confidence interval: $0.3 \%$ to $2.5 \%, p=0.006$, unpaired $t$ test) (Fig. 3, $b$ ). In the control rings, the $\mathrm{EC}_{50}$ was $-7.02 \pm$ $0.07 \log \mathrm{mol} / \mathrm{L}$. After UW treatment, the $\mathrm{EC}_{50}$ value was not changed $(-7.13 \pm 0.04 \log \mathrm{mol} / \mathrm{L}, p=0.3)$.

Contractility of coronary artery after exposure to UW. Under normothermia, exposure to UW significantly reduced the degree of contraction induced by U46619 (30 nmol/L). In the BK experiments, the contraction was $11.4 \pm 1.0 \mathrm{gm}$, and it was reduced to $6.0 \pm 1.9$ gm by the UW exposure $(95 \%$ confidence interval: -0.1 to $0.9 \mathrm{gm}, p=0.01$, paired $t$ test, Fig. 4, a). Similarly, in A23187 experiments, contraction was $7.1 \pm 1.3 \mathrm{gm}$ compared with $10.0 \pm 0.6 \mathrm{gm}(95 \%$ confidence interval: -0.2 to $0.9 \mathrm{gm}, p=0.03$, paired $t$ test) in the control rings (Fig. 4, $a$ ). 

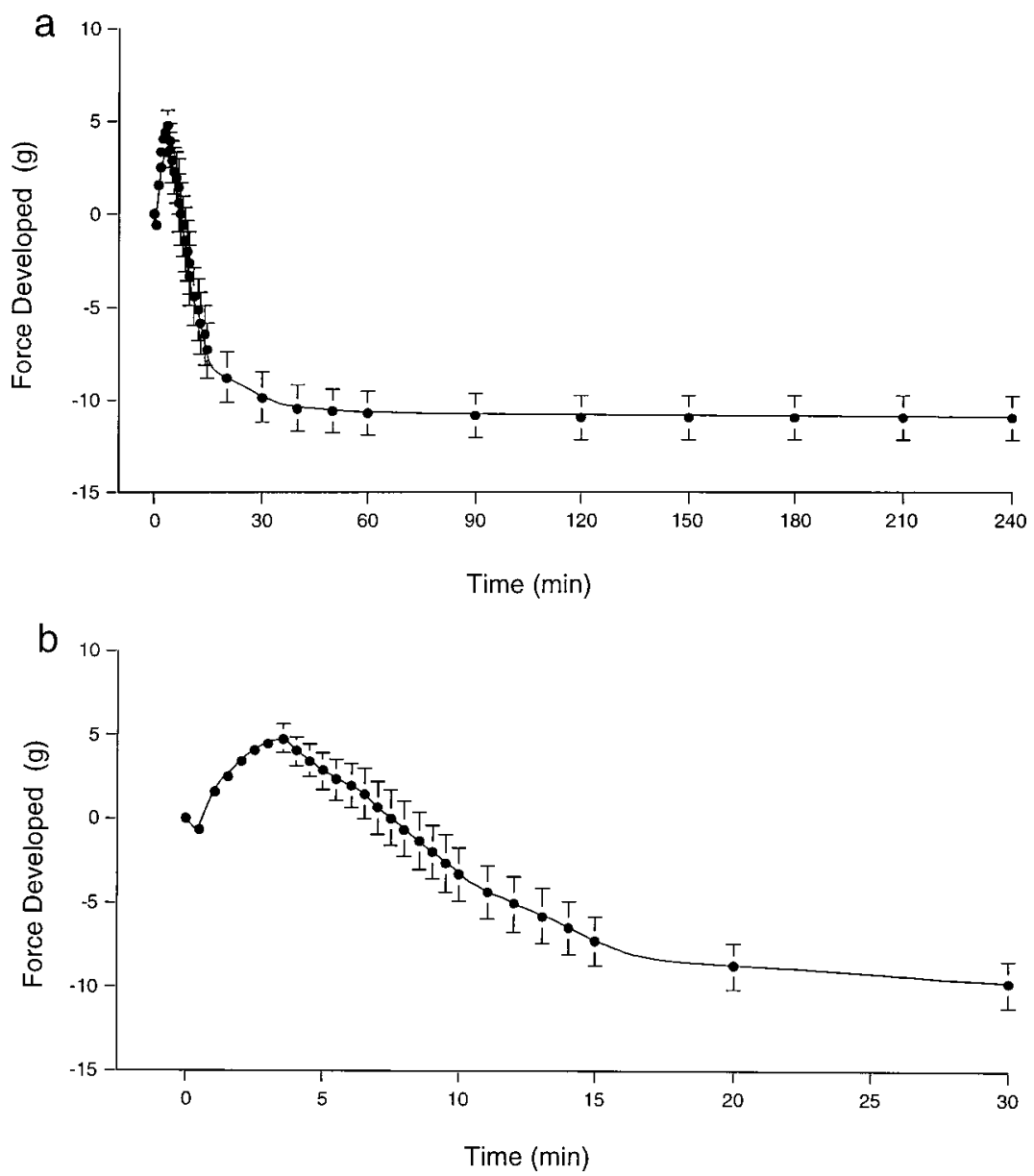

Fig. 1. Isometric force developed in porcine coronary artery ring segments during 4-hour period of incubation with UW (a). Symbols represent data averaged from five coronary rings. Vertical error bars are 1 standard error of the mean. The initial force change during the first 30 minutes is shown in an enlarged time scale in b. The figure shows that there are two phases (the initial contraction and the subsequent relaxation) in terms of force change in coronary arteries during exposure to UW.

After exposure to UW under normothermia, the maximal contraction to U46619 was also reduced (Fig. 4, $b$ ). The maximal contraction to U46619 was reduced to $10.0 \pm 1.4 \mathrm{gm}$, compared with $14.2 \pm 1.0$ gm in the control rings $(n=10$ in each group, $95 \%$ confidence interval: 0.15 to $1.5 \mathrm{gm}, p=0.02$, unpaired $t$ test). The $\mathrm{EC}_{50}$ was 5.1-fold higher in the rings after exposure to UW $(-7.02 \pm 0.11$ vs $-7.73 \pm$ $0.12 \log \mathrm{mol} / \mathrm{L}, 95 \%$ confidence interval: 0.40 to 4.0 $\mathrm{gm}, p=0.0003)$.

In contrast, after cold storage with UW, the contraction to $\mathrm{U} 46619(30 \mathrm{nmol} / \mathrm{L})$ did not change $(11.4 \pm 1.0 \mathrm{gm}, n=9$ vs $9.6 \pm 1.1 \mathrm{gm}, n=9, p=0.3$, in BK experiments and $12.0 \pm 0.8 \mathrm{gm}, n=15$ vs $10.4 \pm 0.9 \mathrm{gm}, n=9, p=0.2$, in A23187 experiments, unpaired $t$ test, Fig. 4,c).

\section{Discussion}

The present study, for the first time, has demonstrated (1) that indomethacin- and L-NNA-resistant endothelium-dependent (EDHF-related) relaxation is reduced by preservation with UW, which is an intrinsic characteristic of the solution, and that this damaging effect is seen in the coronary artery after normothermic and normoxic exposure, as well as after hypothermic and hypoxic storage; (2) that the coronary tone during exposure to UW is biphasic-an initial phase with a transient increase and subsequent phase with a decrease; and (3) that the exposure to UW may impair the coronary smooth muscle contractility but this is protected by hypothermia.

The findings from the present study, in addition to 


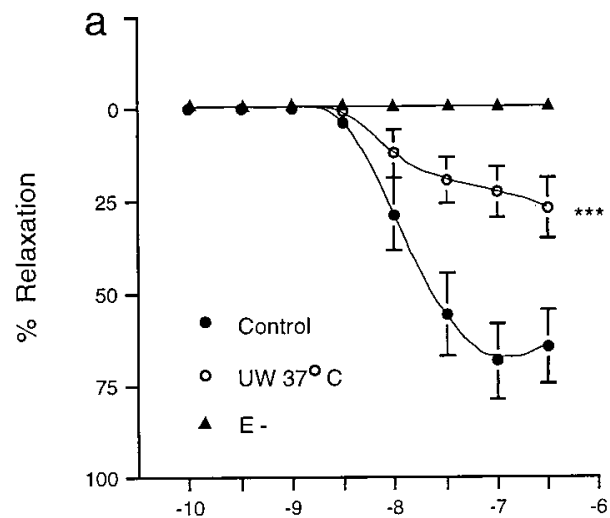

Bradykinin $\log M$

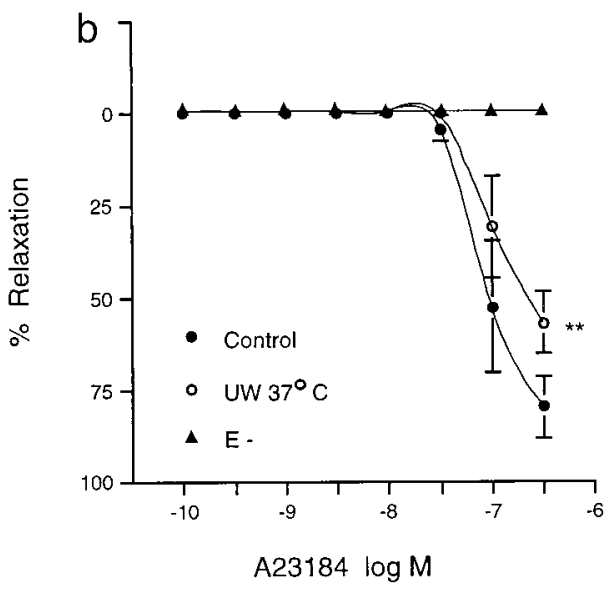

Fig. 2. Mean concentration (-log mol/L) and relaxation (percent of contraction by $\mathrm{U} 4661930 \mathrm{nmol} / \mathrm{L}$ ) curves for bradykinin (a) or A23187 (b) in the coronary arteries exposed to UW for 4 hours at $37^{\circ} \mathrm{C}$. Vertical error bars are 1 standard error of the mean. Control $(n=7)$ : in the presence of indomethacin $(7 \mu \mathrm{mol} / \mathrm{L})$ and $\mathrm{L}-\mathrm{NNA}(300 \mu \mathrm{mol} / \mathrm{L})$ in physiologic solution; $\mathrm{O}, \mathrm{UW} 37^{\circ} \mathrm{C}$ : after exposure to $\mathrm{UW}$ at $37^{\circ} \mathrm{C}$ for 4 hours ( $n=7$ in each group) in the presence of indomethacin $(7 \mu \mathrm{mol} / \mathrm{L})$ and $\mathrm{L}-\mathrm{NNA}(300 \mu \mathrm{mol} / \mathrm{L})$; E-: response to bradykinin or A23187 in endothelium-denuded coronary arteries $(n=4)$. ** $p=0.01$; $* * * p=0.001$ compared with the control (paired $t$ test).
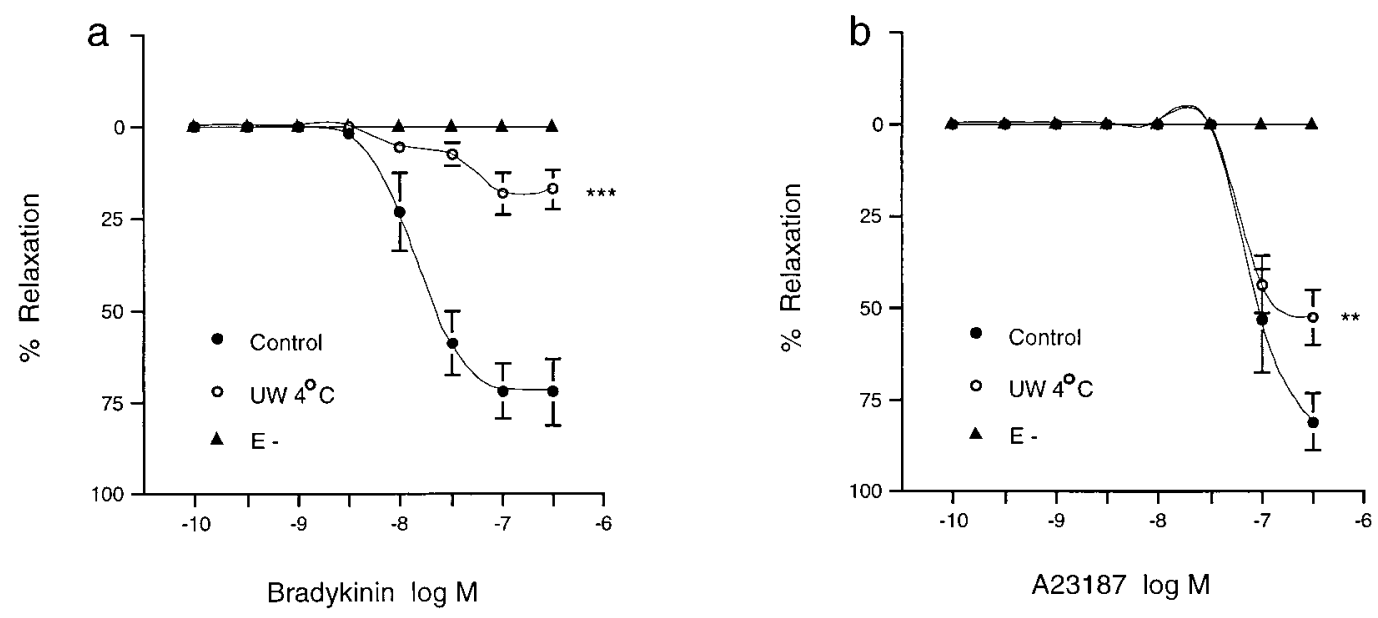

Fig. 3. Mean concentration (-log mol/L) and relaxation (percent of contraction by $\mathrm{U} 4661930 \mathrm{nmol} / \mathrm{L}$ ) curves for bradykinin (a) or A23187 (b) in the coronary arteries exposed to UW for 4 hours at $4^{\circ} \mathrm{C}$. Vertical error bars are 1 standard error of the mean. Control ( $n=7$ for $\mathrm{BK}$ and $n=15$ for A23187), in the presence of indomethacin $(7 \mu \mathrm{mol} / \mathrm{L})$ and L-NNA $(300 \mu \mathrm{mol} / \mathrm{L})$ in physiologic solution; $\bigcirc, \mathrm{UW} 4^{\circ} \mathrm{C}$ : after exposure to $\mathrm{UW}$ at $4^{\circ} \mathrm{C}$ for 4 hours $(n=9$ in each group) in the presence of indomethacin $(7 \mu \mathrm{mol} / \mathrm{L})$ and L-NNA $(300 \mu \mathrm{mol} / \mathrm{L})$; E-: response to bradykinin or A23187 in endothelium-denuded coronary arteries $(n=4) .{ }^{* *} p=0.006 ;{ }^{* * *} p<0.001$ compared with the control (unpaired $t$ test).

the previous ones, may reveal a new mechanism for endothelial dysfunction after exposure to UW and therefore have clinical implications in transplantation surgery.

Coronary endothelial function. Endothelial cells derive three major EDRFs. Unlike EDNO and
$\mathrm{PGI}_{2}$, which have been well studied, the nature of EDHF has not been finally identified, although most recently the cytochrome P450-monooxygenase metabolite of arachidonic acid has been suggested to be EDHF. ${ }^{17}$ EDHF induces vascular smooth muscle relaxation via hyperpolarization of the smooth mus- 

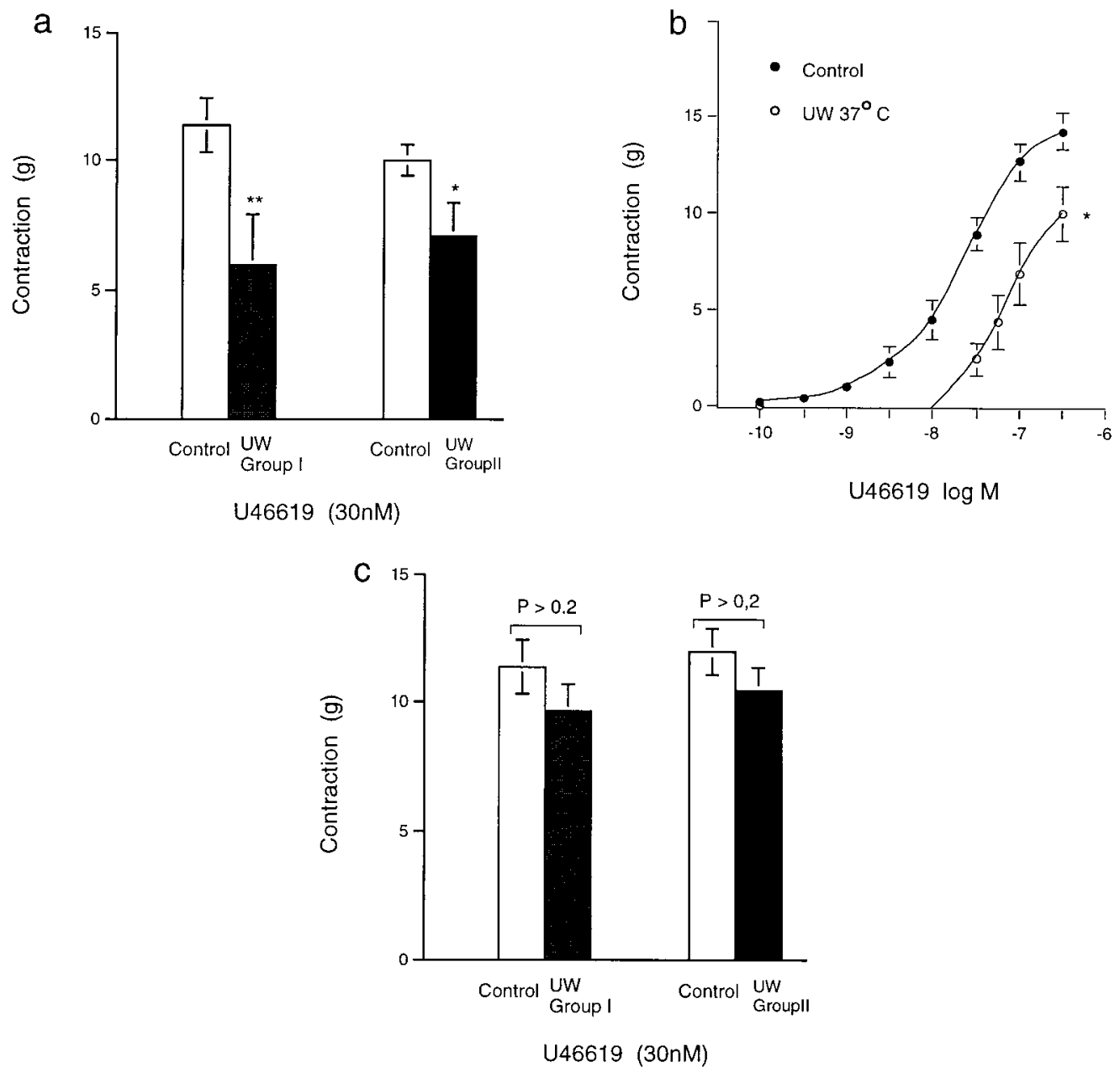

Fig. 4. Contraction force (gm) to $\mathrm{U} 46619$ (30 nmol/L) (a) and mean concentration ( -10 to $-6.5 \mathrm{log}$ $\mathrm{mol} / \mathrm{L}$ )-contraction (gm) curves to U46619 (b) after incubation with $\mathrm{UW}$ at $37^{\circ} \mathrm{C}$ for 4 hours. a, Control: before the incubation; UW group I: in bradykinin experiments $(n=7)$; UW group II: in A23187 experiments $(n=7)$. b, $\mathbf{O}$, Control, in physiologic solution, $n=10 ; \bigcirc$, after incubation with $\mathrm{UW}$ at $37^{\circ} \mathrm{C}$ for 4 hours $(n=10) .{ }^{*} p=0.02$ (unpaired $t$ test). c, Contraction force $(\mathrm{gm})$ to U46619 $(30 \mathrm{nmol} / \mathrm{L})$ after incubation with UW at $4^{\circ} \mathrm{C}$ for 4 hours. Control: $n=6$ for BK (group I, $n=9$ ) and $n=15$ for A23187 (group II, $n=9$ ).

cle cells, ${ }^{6,9,18,19}$ which may involve potassium $\left(\mathrm{K}^{+}\right)$ channels. ${ }^{6,9,20,21}$ In contrast, EDNO relaxes blood vessels through the cyclic guanosine monophosphate pathway. However, all of these EDRFs are released in response to the increase of intracellular (cytosolic free) calcium concentration in the endothelial cell. ${ }^{6,17}$

Although the exact role of EDHF in regulating vascular tone and the development of vascular diseases is still unclear, there is evidence that EDNO and EDHF are two primary mechanisms of endo- thelium-dependent relaxation. $^{21}$ In the present study, the EDHF stimuli BK and A23187 induced $68.7 \%$ and $79.9 \%$ relaxation, respectively. This suggests the role of EDHF in regulating the coronary tone at least under the stimulated condition when the EDNO mechanism is blocked. Studies also show that EDHF may back up or enhance the relaxing action of EDNO, particularly when EDNO-mediated relaxation is impaired, ${ }^{21}$ as seen in some pathologic states such as hypercholesterolemia, hypertension, and diabetes mellitus. ${ }^{21}$ It may be true that in 
the coronary circulation during ischemia and reperfusion period, when the EDNO mechanism is impaired, ${ }^{10}$ the EDHF mechanism may play an important role in regulating the coronary circulation and in developing vasculopathy.

Coronary tone during UW incubation. $\mathrm{K}^{+}$is a depolarizing agent and a strong vasoconstrictor. ${ }^{3,6,22,23}$ There is concern that UW may constrict vessels during the exposure (preservation) period. Our present study provides evidence that during the incubation with UW, although initially the coronary artery contracts, the contraction period is very short (during the first 7.5 minutes) (see Fig 1). After 8 minutes the vessel started to relax and the relaxation persisted for the rest of the incubation period (4 hours). Therefore, despite its extremely high $\mathrm{K}^{+}$ concentration, the coronary artery is in a relaxant rather than a contraction state during the heart preservation period with UW. The vascular tone is determined by the interaction among the effects of the individual components in the solution. As we demonstrated previously, in the St. Thomas' Hospital cardioplegic solution, the vascular tone is slightly reduced although it is a hyperkalemic solution. ${ }^{3,4}$ This effect is probably related to components such as magnesium $\left(\mathrm{Mg}^{2+}\right)$ or adenosine in the solution. In fact, UW contains a $5 \mathrm{mEq} / \mathrm{L}$ concentration of $\mathrm{Mg}^{2+}$. Studies have demonstrated that high concentrations of $\mathrm{Mg}^{2+}$ depress the sensitivity of vessels to $\mathrm{K}^{+24}$ and that $\mathrm{Mg}^{2+}$ induces smooth muscle relaxation $^{25}$ because of the decreases of the intracellular $\mathrm{Ca}^{2+}$ concentration. ${ }^{24}$

It is therefore logical to hypothesize that the vasorelaxant effect of $\mathrm{UW}$ is related to its $\mathrm{Mg}^{2+}$ content, although the effect of other components such as adenosine may also be involved.

EDHF-related relaxation after UW exposure. The effect of UW on EDHF-mediated endothelial function is shown by the reduction of the relaxation induced by either BK or A23187 after exposure to UW for 4 hours under normothermia. In the BK experiments, the relaxation was reduced to $32.1 \%$ ( $p=0.001)$, whereas the A23187-induced relaxation was reduced to $56.9 \%(p=0.01)$. The alteration of the EDHF-mediated relaxation is also shown by the increased $\mathrm{EC}_{50}$ to $\mathrm{A} 23187$ (1.9-fold higher, $p<$ $0.05)$.

Effect of hypothermic storage on EDHF-related relaxation. UW is designed for cold storage of the organ. It has been shown that temperature is an important factor when UW is used for the preservation of the rat heart. ${ }^{12}$ The coronary vascular resistance significantly increased when UW was used at $15^{\circ}$ or $20^{\circ} \mathrm{C}$, which implies an impaired endothelium-dependent relaxation. However, the increase was not significant at $4^{\circ}$ or $10^{\circ} \mathrm{C}$. To study whether the impaired EDHF-mediated relaxation occurs during hypothermic and hypoxic storage, the second protocol of the experiment was designed in our study. The coronary artery was stored in cold UW $\left(4^{\circ} \mathrm{C}\right)$ for 4 hours without oxygenation, a situation similar to the clinical setting. Our results clearly demonstrate that after cold storage with UW, EDHF-mediated relaxation is also impaired. Therefore we believe that the influence of UW on the coronary EDHF-mediated relaxation exists in the clinical setting in which the organ is stored in cold UW.

The possible mechanism of the impairment of the relaxation. We have previously demonstrated that after exposure to hyperkalemia, the reduced EDHF-mediated relaxation is related to two mechanisms. ${ }^{6,9}$ First, the prolonged membrane hyperpolarization of the smooth muscle affects the EDHF-mediated hyperpolarization, which is linked with the relaxation. Second, the exposure to hyperkalemia affects $\mathrm{K}^{+}$channels, particularly calcium-activated $\mathrm{K}^{+}$channels, which is related to the EDHF-mediated hyperpolarization in the coronary artery. Since UW contains an extremely high concentration of $\mathrm{K}^{+}$, it is most likely that the reduced EDHF-mediated relaxation after exposure to UW is also related to the above mechanisms.

Fig. 5 shows a proposal for the mechanism. UW, as a hyperkalemic solution, reduces the EDHF-related relaxation. This is probably the result of two mechanisms. ${ }^{6}$ First, it inhibits $\mathrm{K}^{+}$ channels, particularly calcium-activated $\mathrm{K}^{+}$channels, similar to the effect of a calcium-activated $\mathrm{K}^{+}$blocker tetraethylammonium. ${ }^{6}$ Second, it may have a prolonged depolarizing effect on the membrane. These actions greatly affect the hyperpolarizing effect of EDHF and, therefore, reduce the EDHF-related relaxation. This impairment of the relaxation is imposed to impaired EDNO mechanism, ${ }^{10}$ enhances coronary endothelial dysfunction, and reduces the reserve of the coronary relaxation.

Contractility of coronary artery after exposure to UW. Under normothermia, after exposure to UW, the contraction to U46619 was reduced (see Fig. 4, $a$ and $b$ ), with a higher $\mathrm{EC}_{50}$ to U46619. This suggests that the coronary artery is still at a partially 


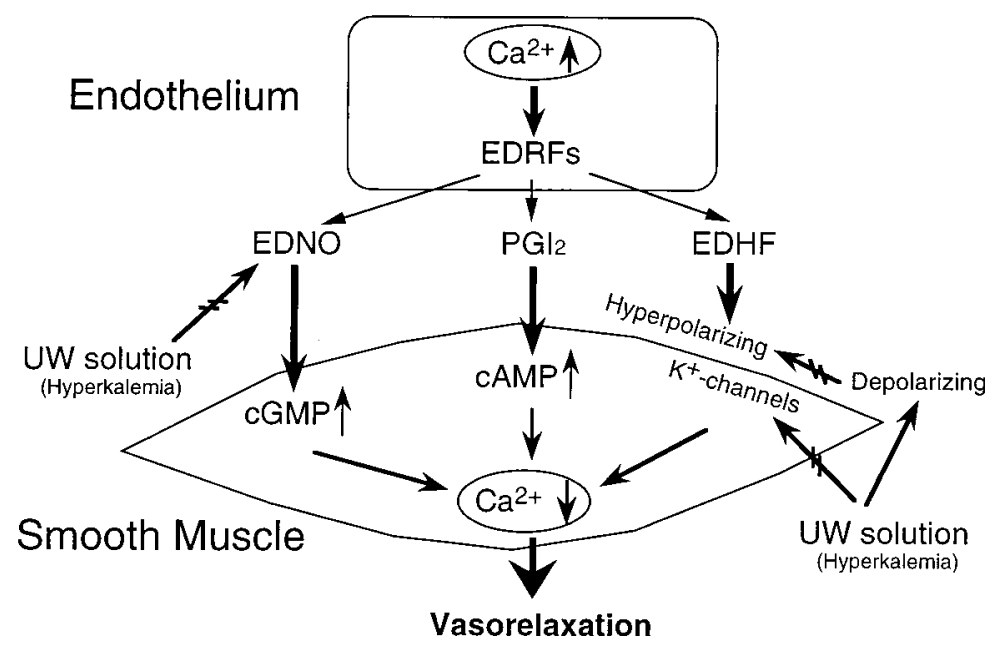

Fig. 5. Schematic diagram describing the possible pathway by which UW alters the endotheliumdependent relaxation. In response to the increase of the intracellular (cytosolic free) calcium level, endothelial cells derive three major EDRFs ( $\mathrm{PGI}_{2}, \mathrm{EDNO}$, and EDHF). These EDRFs decrease the intracellular calcium concentration in the smooth muscle cell through different mechanisms and ultimately relax the smooth muscle cell. The mechanism of EDHF-mediated relaxation is to hyperpolarize the membrane and to open $\mathrm{K}$ channels. Subsequently, the voltage-operated $\mathrm{Ca}^{2+}$ channels are inhibited and therefore the $\mathrm{Ca}^{2+}$ influx is reduced and this causes relaxation. Exposure to UW reduces the EDNO release (reference 10) and the EDHF-mediated relaxation (the present study). As a hyperkalemic solution, the mechanism of the reduced EDHF-related relaxation is likely caused by the change of $\mathrm{K}$ channels and the prolonged depolarization of the membrane, as suggested for the mechanism by hyperkalemia. ${ }^{6}$

plegic status immediately after exposure to UW. However, the contractility of the smooth muscle was preserved after cold storage with UW. This is shown by the result that the contraction by U46619 (30 $\mathrm{nmol} / \mathrm{L}$ ) after cold storage with UW was similar to that in the control group.

In summary, exposure to UW at either $37^{\circ}$ or $4^{\circ} \mathrm{C}$ impairs the EDHF-mediated endothelium-dependent relaxation, although cold storage with UW may protect the contractility of the coronary smooth muscle. Taken together with the previous findings that UW impairs the EDNO release,${ }^{10}$ the preservation of coronary artery with UW impairs the endothelial reserve for the endothelium-dependent relaxation.

Clinical implications. The present study suggests that during incubation with UW the coronary tone is reduced and therefore no vasoconstrictor effect is seen in this period. However, during reperfusion (after incubation) the EDHFmediated relaxation is significantly impaired. The impairment of the EDHF-mediated relaxation reduces the functional reserve of the endothelium-dependent relaxation and therefore may impair myocardial perfusion.

In addition, because endothelium also plays an important role in antiplatelet aggregation and prevention of atherosclerosis, ${ }^{26-28}$ the endothelial dysfunction observed in the present study may have an adverse effect on the long-term results of heart or other organ transplantation. In fact, in cardiac allografts preserved in UW, the incidence of late graft atherosclerosis has been twice as high as in grafts preserved in Stanford solution. ${ }^{11}$

The findings from the present study may provide some insight into the cause of the higher atherosclerotic incidence after heart transplantation using UW and open a new area in terms of better preservation of coronary or other vascular endothelium for organ transplantation by adjusting the concentration of $\mathrm{K}^{+28}$ or other components. However, the impact of impaired EDHF on the development of posttransplantation atherosclerosis needs to be further studied. 


\section{REFERENCES}

1. Saldanha C, Hearse DJ. Coronary vascular responsiveness to 5-hydroxytryptamine before and after infusion of hyperkalemic crystalloid cardioplegic solution in the rat heart: possible evidence of endothelial damage. J Thorac Cardiovasc Surg 1989;98:783-7.

2. Evora PRB, Pearson PJ, Schaff HV. Crystalloid cardioplegia and hypothermia do not impair endothelium-dependent relaxation or damage vascular smooth muscle of epicardial coronary arteries. J Thorac Cardiovasc Surg 1992;104:136574.

3. He G-W, Yang C-Q, Wilson GJ, Rebeyka IM. Tolerance of coronary endothelium and smooth muscle to hyperkalemia. Ann Thorac Surg 1994;57:682-8.

4. He G-W, Yang C-Q, Rebeyka IM, Wilson GJ. Effect of neonatal endothelium and smooth muscle to hyperkalemic cardioplegic solutions. J Heart Lung Transplant 1995;14:92101.

5. Nilsson FN, Miller VM, Vanhoutte PM, McGregor CGA. Methods of cardiac preservation alter the function of the endothelium in porcine coronary arteries. J Thorac Cardiovasc Surg 1991;102:923-30.

6. He G-W, Yang C-Q. Graier WF, Yang J-A. Hyperkalemia alters EDHF-mediated hyperpolarization and relaxation in coronary arteries. Am J Physiol 1996;271:H760-7.

7. He G-W, Yang C-Q. Hyperkalemia alters endotheliumdependent relaxation through non-nitric oxide and noncyclooxygenase pathway: a mechanism for coronary dysfunction due to cardioplegia. Ann Thorac Surg 1996;61:1394-9.

8. Dagenais F, Buluran J, Cartier R. In vitro endothelial dysfunction after cold storage: comparison with various preservative solutions. Ann Chir 1995;49:700-5.

9. He G-W, Yang C-Q, Yang J-A. Depolarizing cardiac arrest and EDHF-mediated hyperpolarization and relaxation in coronary arteries: the effect and mechanism. J Thorac Cardiovasc Surg 1997;113:932-41.

10. Pearl JM, Laks H, Drinkwater DC, et al. Loss of endothelium-dependent vasodilatation and nitric oxide release after myocardial protection with University of Wisconsin solution. J Thorac Cardiovasc Surg 1994;107:257-64.

11. Drinkwater D, Rudis E, Laks H, et al. UW versus Stanford cardioplegia and the development of allograft coronary arterial vasculopathy [Abstract]. J Heart Lung Transplant 1994; 13:S47:64.

12. Mankad PS, Slavik Z, Yacoub M. Endothelial dysfunction caused by University of Wisconsin preservation solution in the rat heart: the importance of temperature. J Thorac Cardiovasc Surg 1992;104:1618-24.

13. Drinkwater DC, Ziv ET, Laks H, et al. Extracellular and standard University of Wisconsin solutions provide equivalent preservation of myocardial function. J Thorac Cardiovasc Surg 1995;110:738-45.

14. He G-W, Buxton B, Rosenfeldt F, Wilson AC, Angus JA.
Weak $\beta$-adrenoceptor-mediated relaxation in the human internal mammary artery. J Thorac Cardiovasc Surg 1989;97: 259-66.

15. He G-W, Yang C-Q. Effects of thromboxane $\mathrm{A}_{2}$ antagonist GR32191B on prostanoid and nonprostanoid receptors in the human internal mammary artery. J Cardiovasc Pharmacol 1995;26:13-9.

16. He G-W. Hyperkalemia-exposure impairs EDHF-mediated endothelial function in the human coronary artery. Ann Thorac Surg 1997;63:84-7.

17. Graier WF, Simecek S, Sturek M. Cytochrome P450 monooxygenase-related signalling of $\mathrm{Ca}^{2+}$ entry in human and bovine endothelial cells. J Physiol 1995;482:259-74.

18. Feletou M, Vanhoutte PM. Endothelium-dependent hyperpolarization of canine coronary smooth muscle. Br J Pharmacol 1988;93:515-24.

19. Chen G, Suzuki H, Weston AH. Acetylcholine releases endothelium-derived hyperpolarizing factor and EDRF from rat blood vessels. Br J Pharmacol 1988;95:1165-74.

20. Scornik, FS, Codina J, Birnbaumer L, Toro L. Modulation of coronary smooth muscle Kca channels by Gs alpha independent of phosphorylation by protein kinase A. Am J Physiol 1993;265(4 pt 2):H1460-5.

21. Cohen RA, Vanhoutte PM. Endothelium-dependent hyperpolarization: beyond nitric oxide and cyclic GMP. Circulation 1995;92:3337-49.

22. He G-W, Yang C-Q. Comparison among arterial grafts and coronary artery: an attempt at functional classification. J Thorac Cardiovasc Surg 1995;109:707-15.

23. He G-W, Yang C-Q, Starr A. An overview of the nature of vasoconstriction in arterial grafts for coronary surgery. Ann Thorac Surg 1995;59:676-83.

24. Skajja K, Forman A, Andersson K-E. Effects of magnesium on isolated human fetal and maternal uteroplacental vessels. Acta Physiol Scand 1990;139:551-9.

25. Barany M, Barany K. Protein phosphorylation during contraction and relaxation. In: Barany M, editors. Biochemistry of smooth muscle contraction. San Diego. Academic Press: 1996. p. 330-1.

26. Shimokawa H, Vanhoutte PM. Impaired endotheliumdependent relaxation to aggregating platelets and related vasoactive substances in porcine coronary arteries in hypercholesterolemia and atherosclerosis. Circ Res 1989;64: 900-14.

27. Shimokawa H, Aarhus LL, Vanhoutte PM. Porcine coronary arteries with regenerated endothelium have a reduced endothelium-dependent responsiveness to aggregating platelets and serotonin. Circ Res 1987;61:256-70.

28. Lee J, Drinkwater D, Laks H, et al. Preservation of endothelium-dependent vasodilation with low-potassium University of Wisconsin solution. J Thorac Cardiovasc Surg 1996;112: 103-10. 